\title{
La co-creación como plataforma estratégica para reposicionar la marca ciudad. Caso de estudio: Medellín, ciudad innovadora
}

Cocreation as a strategic platform to reposition the city-brand.

Case study: Medellín, an innovative city

Daniela Alejandra Gordillo Rojas Estudiante de Publicidad Internacional, Universidad Sergio Arboleda (Colombia). Investigadora adscrita al semillero Epika, grupo de investigación Creategia aleja.gordillo47@gmail.com

Artículo de investigación

Fecha de recepción: Julio 14 de 2015• Fecha de aprobación: Noviembre 24 de 2015

\section{RESUMEN}

Identificando el crecimiento de fenómenos sociales que hoy logran modificar la sociedad, se desarrolló una investigación enfocada en la cocreación como innovación social que toma relevancia en el desarrollo de la cultura urbana, partiendo de la pregunta: ¿cómo se articula la cocreación dentro de una estrategia de marca-ciudad para lograr transformar su posicionamiento? Para responderla, se establece una base teórica y se analizan casos reportados respecto al tema, para posteriormente elegir a Medellín y realizar un seguimiento documental en línea de su estrategia como marca-ciudad a partir de la plataforma de cocreación ciudadana MiMedellín. org. Se analiza su aporte a crear un posicionamiento definido y se concluye identificando cómo la cocreación trasciende y logra ser eje transversal de toda la estrategia de ciudad, aportando significativamente a su construcción como marca, transformando positivamente su posicionamiento a nivel local e internacional.

Palabras clave: innovación social, cocreación, marca-ciudad, posicionamiento, desarrollo urbano. 


\section{AbSTRACT}

Identifying the current growing of social phenomenons that get to modify society, it was developed a reseach focused on cocreation as social innovation that takes relevance in urban culture construction, based on the question: How cocreation is articulated inside a city-brand strategy in order to transform its positioning? To answer, it was established a theorical base, in the same way, reported cases about the topic were analyzed to finally choose Medellín to make an online documental tracking to its strategy as a city-brand from the social cocreation platform MiMiedellin.org. It is analyzed its contribution to create a defined positioning, and finally it is identified how cocreation trascends and achieves to become transverse axis of all the city strategy, contributing significantly to its construction as a Brand, and positively transforming its position locally and internationally.

Keywords: Social innovation, cocreation, city-brand, positioning, urban development.

\section{Naturaleza Y ORIGEN DEL ARTÍCUlo}

El presente artículo se desarrolla como fruto de una labor de investigación desarrollada por más de un año en el semillero Epika, el cual hace parte del grupo de investigación Creategia del programa de Publicidad Internacional de la Universidad Sergio Arboleda (Colombia), perteneciente a la plataforma ScienTI de Colciencias con una clasificación como grupo $\mathrm{D}$, adscrito al área de conocimiento en Ciencias Sociales, específicamente en Periodismo y Comunicaciones.

Teniendo en cuenta que uno de los proyectos del semillero es el Observatorio de Tendencias y Estilos de Vida, que tiene como objetivo hacer un aporte a la formación de investigadores en el área, logrando aportes en consecuencia a la industria publicitaria, la autora que hace parte de la división de innovación del mismo, ha desarrollado este artículo de investigación como un ejercicio reflexivo bajo la línea de investigación en Estética, Cultura y Consumo, en donde se enfoca en profundizar y analizar el impacto de la cocreación como innovación social en el establecimiento de una estrategia de marca y especialmente su posicionamiento, lo cual da cuenta 
de cómo las prácticas innovadoras de la nueva era están transformando los distintos escenarios de comunicación y afectando así también a las marcas y por ende, representan transformaciones para la industria publicitaria.

Este artículo representa además la opción de trabajo de grado elegida por la autora para cumplir con el requisito y así obtener, posterior a su aprobación para publicación, su titulación como Publicista Internacional, otorgado por la Universidad Sergio Arboleda.

\section{INTRODUCCIÓN}

$\mathrm{Al}$ igual que los seres humanos, las marcas, definidas como la representación mental, el conjunto de impresiones o incluso los valores que la gente relaciona o asocia a un determinado sujeto-entidad, organización o empresa (Tinto, 2008), también se han visto obligadas a evolucionar por el impulso de la sociedad. La apertura de las comunicaciones, la era de la web 2.0, entre otros factores, han conducido al desarrollo de nuevas dinámicas de interacción entre marca y consumidor, pasando desde un modelo de una sola vía, donde el anunciante tenía un papel único como emisor de mensajes y proveedor de productos; posteriormente la apertura bidireccional, donde el consumidor además de ser receptor de estos mensajes, podía responder de vuelta, hasta que finalmente se encuentra el panorama actual. Ahora, la relación no solo es bidireccional, pues es tal la apertura de canales y las disposiciones de información, que tanto anunciantes, como público objetivo, tienen un rol multipropósito, que les permite hacer las veces de emisores, receptores, productores, creadores y compradores casi de forma simultánea en una sola interacción.

De este modo, se hace aún más evidente la increíble velocidad y facilidad con que se transforman las dinámicas culturales del entorno global e incluso, a nivel local, ya que la era digital, con su democratización de la información, ha traído como una de sus principales consecuencias un mundo marcado por la apertura de las comunicaciones que permite a los usuarios tomar roles relevantes, que además de brindar una experiencia de empoderamiento personal, aporta un valor que trasciende y afecta a toda una sociedad, lo cual habilita toda una gama de oportunidades 
únicas para el mercado, que de ser identificadas, pueden ser aprovechadas por las marcas e instituciones.

En este contexto, las personas buscan soluciones cada vez más efectivas y eficientes ante la multiplicidad de problemáticas sociales, en tanto los procesos y lineamientos tradicionales ya no responden a las cambiantes necesidades. Es entonces donde se hace relevante el concepto de innovación social, no solo como un recurso, sino más como una necesidad imperativa para todo contenido, iniciativa o propuesta que se presente como respuesta a las demandas de la sociedad y, por ende, se ha convertido en una herramienta de gran valor para las estrategias corporativas y de comunicación de marca.

De modo general, la innovación se define como "el proceso en el cual a partir de una idea, invención o reconocimiento de una necesidad, se desarrolla un producto, técnica o servicio útil hasta que sea comercialmente aceptado" (Escorsa, 1997). Mas hay que resaltar que tal como lo afirma Geoff Mulgan, CEO de Nesta -el más importante laboratorio de innovación- dentro de sus diversos tipos, la rama social de la innovación es requerida hoy más que nunca para lograr transformaciones en las políticas públicas, desarrollo de las comunidades y de las ciudades, ya que es esta tal vez, la herramienta más idónea para brindar una visión diferente, que al surgir como una respuesta a una necesidad real y relevante identificada en la sociedad, puede aportar nuevos elementos al diseñar una solución que contemple e involucre a todos los actores.

Es entonces cuando aparecen espacios de intermediación denominados plataformas digitales, que contribuyen al desarrollo comercial y económico organizando la conexión entre la oferta y la demanda al tener un rol como intermediarios que les permite recopilar un gran número de datos sobre las relaciones entre los dos lados del mercado, desarrollando un profundo conocimiento de los modos de consumo y los hábitos (Neutralidad de las plataformas, 2014). Consientes de este panorama, las marcas han desarrollado, por sí mismas, nuevas plataformas que permiten a los usuarios poner en práctica estas nuevas interacciones mucho más abiertas y participativas, pero de modo tal que al mismo tiempo les generen valor agregado, es decir, en lugar de permitir que esta pérdida de control sobre las dinámicas 
de comunicación las afecte negativamente, descubrieron y comprendieron la posibilidad que tenían de aprovechar la situación al crear y otorgar ellos mismos estos espacios de libertad para sus usuarios, de modo que puedan observar de cerca estas interacciones, logrando realimentarse de dicho proceso, y a partir de allí, reinventarse y fortalecerse para mantener relevancia y hacer sostenible un vínculo con sus clientes a lo largo del tiempo.

Dentro de estos nuevos espacios de interacción habilitados por las marcas, aparecen las que son reconocidas como plataformas de cocreación. Estos espacios empoderan a los consumidores, permitiéndoles intervenir, al menos con ideas, en sus procesos de creación y desarrollo de producto, entablando un diálogo directo que genera mucha más cercanía y confianza al poner a su disposición distintas herramientas y lineamientos, a partir de los cuales los consumidores revelan por voluntad propia los imaginarios, expectativas e ideas de todo tipo que inundan sus mentes alrededor de un producto o servicio, de modo tal que habilitan a las marcas para desarrollarse a la medida de los verdaderos deseos y necesidades de las personas. La primera definición de cocreación, categorizada como innovación social, es citada por Parente (2013) y fue hecha por Neeli y Leone (1979):

Una opción para la creación de productos que incluye a todos los involucrados: clientes, proveedores, actores internos entre otros. Cocreación no es la manera de testearla efectividad de un productooculminarcon sucreación, cocreación incluye la creación de un producto desde sus inicios con la guía de un coach, generalmente parte de la empresa, que ayuda a concretar las ideas y a organizar el grupo (tomado de http://blogs.portafolio.co/los-aparentes-negocios-internacionales/ innovacion-y-mas-innovacion-que-tal-co-creacion/).

Esta práctica genera un mayor valor mutuo a partir del trabajo y la interacción conjunta, mas requiere de un verdadero esfuerzo y compromiso en el cual las empresas deben dejar de ver al individuo como un receptor pasivo e involucrarlo activamente en la definición y entrega de valor. Según Ramaswamy y Gouillart (2010), no se trata de "pensar fuera de la caja", se trata de "transformar la caja", y para lograrlo definen cinco componentes clave: una comunidad, una plataforma de participación, la expansión constante de las interacciones, nuevas experiencias para que todas las 
personas salgan ganando y un nuevo valor para quien crea la comunidad. Se trata entonces de una nueva era, donde la inteligencia colectiva logra revelar soluciones disruptivas con un enorme potencial para causar un impacto significativo, de modo que incluso se logra llevar el consumo a una dimensión productiva.

Con todo lo anterior, podría decirse que existe un escenario idóneo para que las marcas consideren la cocreación como una herramienta de gran viabilidad para sus estrategias, pues en definitiva, se trata de una experiencia que genera un nuevo valor para ambas partes y que constituye un insumo de proyectos creativos de distinta índole, por lo cual, en el ámbito del desarrollo urbano, cumple los mismos objetivos, pues, "¿quiénes están más capacitados para hablar de los elementos esenciales de un lugar que sus propios habitantes? ¿Quiénes conocen mejor sus historias, sus atributos y formas de vida?" (Las Salinas, 2015).

Se trata entonces de entender que el potencial de un fenómeno como este no está limitado al mundo de las marcas empresariales de bienes y servicios, pues es sabido que también es posible catalogar efectivamente tanto a países y ciudades como marcas activas; así lo señala Puig (2009), y explica precisamente el gran valor que se imprime a un territorio al ser pensado y diseñado como marca:

Confiar en una apuesta: en una marca. Una marca que crea ciudad de valor espléndida, impulsada por un equipo público, con el consenso entusiasta de los ciudadanos y sus organizaciones plurales. La marca es la propia ciudad, el estilo con el que queremos vivir y convivir. Una marca pública es una apuesta por avanzar en humanidad esperanzada. Una marca es ciudad con futuro compartido (pp. 15-16).

Entendida de esa manera, una ciudad-marca está en perfectas condiciones para aprovechar herramientas como la cocreación e incluirlas como parte de su estrategia y de acuerdo a sus propios objetivos, inmersos en su dinámica de mercado particular, pues en la medida que se reconoce que este es tal vez, uno de los escenarios donde la participación activa de la sociedad, en cooperación con la marca-ciudad, puede tener un valor más tangible, resulta una temática de gran interés pues sus efectos se verán reflejados directamente sobre la calidad de vida de los individuos y bajo este último 
planteamiento, parte un ejercicio reflexivo que basado en la observación y el estudio de casos concretos, contrasta también con la investigación de fuentes relevantes, de modo que contribuya a comprender y analizar el impacto -actual y potencial- que ejercen las plataformas de cocreación en este tipo de marcas ciudad, siendo para ellas tan particularmente determinante el factor social.

Vale la pena entonces hacer un breve análisis de las variables que conllevan a las ciudades a plantearse este tipo de retos. En primer lugar, hay que entender que las ciudades son sistemas de muchas partes, que interactúan entre sí y con el entorno por una multiplicidad de canales y de maneras difícilmente predecibles, por lo cual es clave accionar procesos de innovación en el sector público (Waissbluth et al., 2014). Además, es importante entender que las ciudades, lo quieran o no, son marcas del dominio público, tal como lo plantea Juan Pablo Rocha, presidente de JWT Colombia -agencia actual del gobierno distrital de Bogotá-, quién explicó en entrevista que, si por definición una marca se reconoce como un conjunto de percepciones y asociaciones mentales comunes para un grupo de individuos, se reconoce entonces que toda ciudad es marca, pues más allá del hecho de ser ampliamente reconocida o no, es percibida con unas características y asociada a conceptos específicos, es decir, cuenta inherentemente con un posicionamiento definido, aun si ese lugar en el imaginario colectivo no fue construido a partir de unos lineamientos estratégicos, ni fruto de un proceso de construcción o branding (2016).

El interés y la necesidad por parte de la ciudades de hacer uso de herramientas innovadoras surge entonces como consecuencia directa del actual fenómeno de movilización global hacia la era de las smart cities -en el marco de la era tecnológica-, donde la imagen de marca de una ciudad o lugar se convierte más que nunca en sinónimo de estado de opinión activo y generado; lo que a su vez implica el reconocimiento de un perfil de ciudadanos activamente involucrados y comprometidos con los procesos de administración, innovación y toma de decisiones. Respondiendo a este escenario la cocreación aparece como un método específico para hacer tangible ese reconocimiento, empoderando a los individuos pertenecientes a diferentes grupos de interés y promoviendo su papel como gestores de cambio hacia un mejor futuro para su propia comunidad (Ransbeeck, 2015). 
Podría decirse entonces, que se trata de una auténtica revolución donde ya no son los estudios de grandes firmas, con predicciones y conclusiones, con porcentajes y márgenes de error, las que direccionan las decisiones de las compañías, sino que ahora ellas pueden establecer un dialogo personal con cada consumidor -con todo lo que eso implica- y obtener de fuentes primarias todas las guías necesarias para avanzar en el mercado de forma mucho más competitiva y relevante.

\section{Metodología}

Para llevar a cabo este proyecto investigativo, se propuso una metodología con enfoque cualitativo, que permitió hacer un abordaje a profundidad de cada una de las variables y categorías de interés, sabiendo que "los métodos cualitativos son los que enfatizan conocer la realidad desde una perspectiva insider, de captar el significado particular que a cada hecho atribuye su propio protagonista, y de contemplar estos elementos como piezas de un conjunto sistemático" (Ruiz, 2012, p. 17). Siendo estas características propias de la metodología, resultó pertinente en la medida que, al ser la cocreación una innovación de tipo social, toda profundización sobre ella debe tener ese factor "social" como un eje, lo cual implica la contemplación de un conjunto de variables en simultáneo que se dan en función de las interacciones de los actores, en el cual los datos surgirán precisamente a partir de las relaciones que se detecten entre las diferentes variables del fenómeno de estudio. Por lo tanto, se esperaba que los instrumentos elegidos bajo esta metodología, y que son explicados más adelante, permitieran hacer una mejor aproximación al marco de referencia al cual se enfrentaron, y con sus datos ricos y profundos se logró hacer una mejor descripción de los hechos (Soler, 1997).

Cabe resaltar además que al ser esta una investigación que aborda un tipo de innovación -como lo es la cocreación-, en cuanto a sus objetivos de conocimiento, tenía un enfoque de tipo exploratorio, con el cual se buscó proveer de una visión aproximada que concluyera en un nivel superior de conocimiento al que se poseía previo al proyecto. Lo anterior, teniendo plena consciencia de la gran dimensión de la temática, por lo que al concluir el proyecto, se esperaba llegar al planteamiento de conclusiones que despertaran el interés entre futuros investigadores que se 
atreviesen a plantear nuevas hipótesis y que permitieran una formulación aún más precisa a investigaciones de mayor rango, de forma tal que se lograra edificar nuevo conocimiento.

En cuanto a los instrumentos de recolección de información a utilizar, se procedió de la siguiente forma: en principio, para sentar una base conceptual sólida, se efectuó una revisión documental de fuentes bibliográficas tanto físicas como digitales, que permitió establecer claridad sobre los conceptos base de la temática a abordar y así reconocer e identificar plenamente la información recabada hasta la fecha por fuentes externas, establecer diferentes perspectivas, etapas de desarrollo e incluso el estado de conocimiento en el cual se encontraba la sociedad al respecto del objeto de estudio. Posteriormente, para la aplicación de este instrumento, se hizo una localización de material de interés, como lo son registros documentales (informes y reseñas escritas principalmente), que representaban fuentes de información importantes acerca de las categorías establecidas -en el marco teórico- de este estudio.

Para continuar el proceso investigativo, se consideró pertinente realizar una etnografía digital, aprovechando el aspecto cultural de esta técnica, según la cual se concibe a internet como un espacio de creación para interactuar y generar una cultura propia (Hine, 2011), lo cual permitió reconocer características fundamentales de la tendencia de cocreación aplicada al desarrollo urbano y analizar cuál había sido su evolución y resultados hasta el momento. Así pues, se tomó en un primer momento las bases de datos ya desarrolladas por el semillero de investigación Epika de la Universidad Sergio Arboleda. Este compendio de datos, con una base de 145 plataformas de cocreación de todo el mundo (registradas hasta la fecha de consulta), permitió tener un primer acercamiento al caso de estudio central -Plataforma MiMedellín- a la vez que permitió al investigador observar la proporción de este tipo específico de cocreación contra otros escenarios y tener un balance sobre la relevancia y evolución de estas prácticas a nivel nacional e internacional.

Al efectuar una profundización enfocada en la plataforma MiMedellín, en la etnografía se consultaron entrevistas como fuentes de segunda mano, realizadas a personas que se han visto directamente relacionadas con la plataforma y el desarrollo de la estrategia 
de la marca-ciudad, estableciendo desde cómo se daba su funcionamiento, hasta analizando los resultados obtenidos a esa fecha de acuerdo a las iniciativas que había logrado promover. Además de indagar sobre la percepción que ha logrado generar entre la ciudadanía con sus acciones, sondeándola con un instrumento de investigación tipo encuesta, enviados vía mail a habitantes de Medellín, con preguntas abiertas y cerradas que permitieron conocer su percepción general sobre la ciudad y también su nivel de conocimiento e interés por la plataforma de cocreación ciudadana. Con toda esta información de base, se efectuó un breve análisis para finalmente desarrollar una reflexión acerca de cómo este tipo de innovaciones lograban contribuir a la construcción de la denominada marca-ciudad, es decir que el indicador para medir el impacto que se tomó como eje, fue respecto al posicionamiento logrado, en este caso para la marca-ciudad Medellín.

Adicionalmente, como complemento de todo lo anterior, se realizó una entrevista en profundidad a Juan Pablo Rocha, actual presidente de J. Walter Thomson, una de las más importantes agencias de publicidad colombiana, quien ha estado involucrado directamente con la implementación de una plataforma de cocreación urbana y tiene amplios conocimientos en cuanto a los procesos necesarios para lograr un posicionamiento de marca, más allá de la naturaleza de la misma. La entrevista fue de tipo semiestructurada, de modo que permitió tanto al investigador como al entrevistado, una mayor flexibilidad que facilitó el desenvolvimiento sobre los temas y recabar una mayor cantidad de información que resultó muy útil para analizar los casos particulares, a la luz de una visión integral de la cocreación como práctica de innovación a partir de la contemplación de las variables pertinentes en este escenario.

Así, se determinó finalmente el verdadero grado de impacto sobre el posicionamiento, que puede ser obtenido por una marca-ciudad al innovar en sus estrategias de desarrollo con este tipo de herramientas. Partiendo del contexto social anteriormente evidenciado y con la base teórica establecida, se planteó la pregunta, ¿cómo se articula la cocreación dentro de una estrategia de marca-ciudad para lograr transformar su posicionamiento? 


\section{EXPOSICIÓN DE LOS RESULTADOS}

Para analizar este fenómeno se partió de las bases de datos del semillero Epika de investigación de la Universidad Sergio Arboleda, donde los investigadores han reportado plataformas de innovación para tres ejes de innovación, entre ellos la co-creación.

De acuerdo a estos datos, dentro de las 145 plataformas de cocreación reportadas, 11 están enfocadas en este ámbito. Cinco de estas funcionan para una o varias ciudades europeas; otras cinco pertenecen a América Latina, de la cuales tres, surgen y operan en Colombia, además existe una con operación de tipo global, pero que también fue concebida originalmente en Colombia, lo cual indica un alto nivel de acogida por parte de este último hacia este tipo específico de iniciativas. De hecho, según estos datos, Colombia podría posicionarse como el país con mayor grado de desarrollo y experiencia en el ámbito de la cocreación urbana dentro de América Latina, lo cual sustentaría el por qué desde allí incluso surge una iniciativa de impacto a escala global en este ámbito.

De acuerdo a estos resultados, obtenidos a partir de las bases de datos del semillero de innovación, se tomó la decisión de enfocar la atención en las plataformas colombianas por su fuerte presencia, entre las tres se identificó a la que presentaba una mayor trayectoria y registro sólido de actividad, lo que concluyó señalando a la plataforma MiMedellín como el caso que permitirá analizar de forma más profunda el impacto que este tipo de iniciativas logran tener en el desarrollo de la ciudad, no solo como territorio, sino específicamente en su posicionamiento de marca.

El sistema de la plataforma fue desarrollado por Ángel Tíjaro, quien también estuvo detrás de proyectos como MiQuito y más recientemente, Bogotá Abierta. El ingreso a MiMedellín se logra a través del sitio web http://www.mimedellin.org/, en donde este proyecto se autodefine como: "una plataforma de cocreación ciudadana en donde las ideas y la inspiración de todos forman parte de la transformación de nuestra ciudad". Esta iniciativa fue ideada y liderada principalmente por la Corporación Ruta N Medellín que fue creada por la alcaldía de Medellín, UNE y EPM. Se concibió así 
en el año 2009, concretamente con un objetivo de posicionamiento que otorgara a Medellín el título de la ciudad más innovadora de América Latina para el año 2021.

El hecho de que Medellín, como ciudad, se preocupe por crear una institución como esta, que tiene a razón de ser un objetivo de construcción y transformación de percepción, demuestra el claro interés de la ciudad por construirse y planificarse, no solo desde el ámbito social urbano, sino que revela la concepción que tienen de la importancia alrededor de generar un posicionamiento positivo establecido alrededor del nombre del territorio, es decir, dotar de valor a la marca-ciudad.

Con este pensamiento, Ruta $\mathrm{N}$ desarrolló la estrategia Medellin Innovation, conocida como El gran pacto por la innovación, el cual se fundamenta en cinco ejes que buscan conectar la ciudad a la innovación en todos los sentidos. Uno de estos ejes es Medellín cocreación ciudadana, a partir del cual nació la plataforma MiMedellín en noviembre de 2013. Desde un primer momento, la plataforma buscó generar un cambio de pensamiento entre los ciudadanos, llevándolos de la crítica a la acción, haciendo que todos se comprometieran con la meta de innovación de la ciudad y juntos construyeran su futuro.

A nivel social, la plataforma pretende elevar los niveles de empoderamiento y participación ciudadana, identificando los talentos de los habitantes de la ciudad y promoviendo el uso de nuevas metodologías para crear y ejecutar iniciativas de alto impacto para Medellín. Para esto, el sistema de cocreación en la plataforma funciona bajo un sistema de temporadas de 75 días, para un total de cuatro al año. En cada una establece una temática específica que con anterioridad ha sido identificada -con ayuda de las personas- como una necesidad relevante para la ciudad. Entonces, todos son invitados a ingresar a la plataforma y dar sus propuestas e ideas al respecto, allí también pueden valorar positiva o negativamente otras ideas, compartirlas en redes sociales o comentar con críticas constructivas. Al final de cada temporada, las 10 ideas con mayor potencial y acogida son públicamente reconocidas, para elegirlas se combinan la acogida entre los demás ciudadanos -likes, shares y comentarios positivos- además de la evaluación de un comité de expertos. 
Por supuesto, el objetivo final es que las ideas no se queden plasmadas en la plataforma, sino que aquellas que demuestren viabilidad lleguen a ser proyectos realizados en beneficio de la ciudad y sus habitantes. De acuerdo con una entrevista realizada para el documental Código Origen (Corporación Ruta N, 2016), consultada como fuente secundaria digital, y que fue realizada a Sury García, generadora de contenidos digitales y gestión de comunidades virtuales de MiMedellín, implementar estas ideas conlleva un proceso de maduración y desarrollo, donde cada organismo correspondiente se encarga de llevar su proceso con el ciudadano. Este no es un elemento menor, pues el hacer tangible la experiencia es vital para lograr mantener la motivación, interés y compromiso de los actuales y futuros participantes del proceso de cocreación.

En cuanto a estos resultados reales, MiMedellín tenía proyectado lograr $10 \mathrm{mil}$ ciudadanos como usuarios activos de la plataforma que generaran más de 3.000 propuestas a diciembre de 2014, mas antes de octubre de ese ańo ya habían superado ambas metas, de tal modo que para enero de 2015 la plataforma contaba con 11.816 usuarios activos y 6.133 ideas. Evidentemente el crecimiento y la acogida de esta iniciativa ha sido absolutamente progresivo y positivo, demostrando el gran impacto social que pueden lograr este tipo de espacios de participación abierta para la ciudadanía.

Para celebrar el exitoso cierre de la primera temporada se realizó una ceremonia de clausura en conjunto con la séptima sesión del Foro Urbano Mundial, donde se reconoció a los ganadores que aportaron ideas sobre cómo darle vida a los bajos de los Puentes del Río Medellín, las cuales fueron incluidas en el Plan de Ordenamiento Territorial de la Alcaldía de Medellín y en el diseño del mega proyecto de Parques del Río, buscando hacer que el Río Medellín sea un eje ambiental y de espacio público. En julio de 2014 la obra Parques del Río fue inaugurada en el marco del evento Festival SonRío, es decir, el paso de ideación a la acción se convirtió en una realidad que validaba el ciclo de cocreación ciudadana, experimentada por primera vez en esta ciudad y marcando un referente también para todo el país.

Así, la plataforma ha tenido un gran impacto en la toma de decisiones como principal canal de gestión de comunicación, siendo el eje para el desarrollo de diferentes 
proyectos que impactan directamente en la vida de la comunidad como lo logrado con Comuna Innova, una iniciativa de Ruta $\mathrm{N}$ que recorre los barrios, reuniendo a sus líderes comunitarios, identificando problemas y buscando soluciones innovadoras brindadas por las universidades o las empresas y apoyadas por los ciudadanos (Ruta $\mathrm{N}, 2014$ ), tejiendo así redes y alianzas para el desarrollo de la ciudad del cual han surgido importantes proyectos como "Comunas con nuevos aires", que benefició cerca de 360 mil habitantes de las comunas 8, 9 y 10, a partir de la recuperación del tranvía y sus espacios aledaños (Secretaría de movilidad, Alcaldía de Medellín, 2014) o también el caso del barrio El Compromiso de la comuna 1, un lugar que hoy se ilumina y deja atrás la inseguridad gracias a la energía alternativa de paneles solares fabricados por los habitantes, bajo la instrucción de la Fundación Litro de Luz y el apoyo de la Agencia Internacional de Desarrollo de los Estados Unidos - USAID y Global Communities (MiMedellín, 2016).

Otro de los casos de éxito de la plataforma es "Hagamos de Laureles el primer barrio amigable con la bicicleta", un reto en el que las 5 ideas ganadoras serán implementadas en la construcción de la ciclo-infraestructura de la fase 1, sector Laureles - Estadio (Ruta N, 2015) y además, las ideas fueron expuestas en el Cuarto Foro Mundial de la Bicicleta 2015, pues los directores de la plataforma son conscientes de la gran importancia de dar reconocimiento a los usuarios que participan de la cocreación, además de motivar a otros a unirse una vez comprueban que la cocreación es una oportunidad real para todos.

Ahora bien, conociendo los resultados generales de penetración y alcance logrados hasta ahora por MiMedellín, es momento de analizar su rol dentro de la estrategia de la ciudad y cómo ha logrado contribuir en la meta del nuevo posicionamiento de la capital antioqueña.

Como fruto de la estrategia continua de transformación originada en el 2009 y consolidada con la creación de Ruta N, para el 2013 Medellín fue ganadora del título como la ciudad más innovadora del mundo en el concurso City of the Year, organizado por The Wall Street Journal y Citigroup. Este sería un indicador de éxito anticipado para la ciudad que planteaba desde su estrategia ganar este título para el 2021. Si bien un único concurso no puede certificar la obtención de un 
posicionamiento generalizado, representa sin duda un primer referente importante, que construye de manera significativa hacia este objetivo, pues tan solo la gran cantidad de ruido mediático que provocó esta noticia, contribuye a modificar los imaginarios alrededor de la marca-ciudad. Al respecto, el Urban Land Institute declaró: las ciudades más innovadoras del mundo ponen en práctica sus visiones, derriban barreras y promueven la colaboración para mejorar la calidad de vida de sus habitantes (...). Pero aún más importante, es la transformación de tipo institucional, que permitió que, con un trabajo conjunto entre gobierno local, empresa privada, organizaciones comunitarias y universidades, Medellín lograra enfrentar la violencia y modernizar la ciudad (Semana.com, 2016).

Por su parte MiMedellín como espacio de cocreación ciudadana estaría contribuyendo en este logro al representar ese perfecto escenario de trabajo conjunto, pues cabe aclarar que la plataforma ha permitido no solo el aporte e interacción entre los ciudadanos del común, sino que además permite generar conexiones con entidades y organizaciones de distintos tipos, que pueden aprovechan los recursos de la herramienta para impulsar iniciativas con impacto social de forma más eficiente. Como ejemplo se tiene el caso de la Secretaría de Movilidad, que en el 2014 se apoyó de MiMedellín para ejecutar un proyecto que buscó, junto con los ciudadanos, soluciones para disminuir el uso del vehículo particular (Alcaldía de Medellín, 2014). Además, los directivos de la plataforma han buscado acercamientos, haciendo invitaciones directas a las comunidades universitarias para unirse a este proyecto (Prensa CES, 2015) y aportar con su conocimiento a la construcción de propuestas aún más sólidas, para facilitar su posterior desarrollo al ser llevadas a la realidad de la ciudad.

Se entiende entonces que todo este escenario es posible gracias a que la ciudad ha afrontado el reto de transformar su cultura, como lo indica Felipe Arboleda -creador de InspiraLabs-, "para lograrlo se tomó como principio la tarea de inspirar a los ciudadanos, orientarlos a través de la formación, narraciones e imaginarios presentes en las calles, a los cuales se aferraban" (2016). Lo que en otras palabras significa que empezaron por cambiar el posicionamiento entre los propios ciudadanos, para después poder proyectarlo de forma sólida y contundente al resto del mundo. 
Lo anterior se pudo evidenciar con un sondeo realizado vía e-mail entre 50 habitantes de Medellín, cuando se les preguntó sobre la visión que tenían de la ciudad y el significado que esta tenía para ellos, la totalidad de las respuestas reflejaron percepciones sumamente positivas, las personas hablan de esta como un lugar tranquilo donde edificar sus familias (70\%), donde se sienten libres y ven progreso y oportunidades $(30 \%)$, además lo identifican con orgullo como su hogar, lo que refleja el arraigado sentido de pertenencia presente entre los habitantes.

A propósito, es importante resaltar que gran parte del éxito alcanzado por proyectos de innovación social de tipo participativo, tal como lo es MiMedellín, se debe en parte a la propia naturaleza y espíritu particular de sus habitantes. De acuerdo con otra de las entrevistadas para el documental Código Origen, Angélica Jaramillo, experta en cultura de innovación, existe el mito fundacional de la colonización antioqueña, que sustenta la gran capacidad de cooperación presentes en esta población y da origen a esa esencia que los hace personas profundamente apegadas a su tierra. Para Jaramillo, Medellín es un pueblo innovador, con una generación donde las herramientas de la innovación han evolucionado y hoy usan mucho más el conocimiento para generar esas transformaciones.

Lo anterior se relaciona con una de las claves de éxito que debe poseer toda estrategia de cocreación urbana, que es el nivel de compromiso, ya que el proceso de cocreación entre un gobierno local y sus grupos de interés, requiere de recursos financieros, humanos y de tiempo. Mientras no hay ningún compromiso para lograr cualquier cosa, no habrá ningún éxito (Ransbeeck, 2015).

En el caso de los medellinenses, claramente este nivel de compromiso se deriva de su gran sentido de pertenencia, tanto así que en el sondeo realizado, cuando se nombró la plataforma y se indagó la predisposición a participar en ella, aunque eran pocos quienes ya lo habían hecho $(10 \%)$, todos se mostraron interesadas en hacerlo y calificaron como "muy importante" (70\%) el impacto que este tipo de iniciativas tiene a la hora de contribuir en la imagen positiva de la ciudad, de modo que hay un interés manifiesto por hacer que su ciudad tenga un posicionamiento favorable y todos están a disposición de contribuir de alguna manera para lograr este objetivo. 
Rastreando otros hechos que evidencien cómo se han dado las dinámicas innovadoras en Medellín, se encontró que para el 2015 la ciudad fue la tercera ciudad más importante de Latinoamérica en innovación, en el ranking mundial elaborado por la agencia global en innovación 2thinknow, y más recientemente, la capital antioqueña fue la ganadora del premio Lee Kuan Yew World City 2016, un galardón que reconoce a aquellas ciudades del mundo que logran transformarse rápidamente (Semana.com, 2016).

Por lo tanto, podría afirmarse a partir de estos resultados que la estrategia de la ciudad ha rendido resultados positivos hasta ahora, y si bien esta estrategia debe continuar trabajándose desde distintos ámbitos y de manera continua, las tácticas implementadas a la fecha, como MiMedellín por ejemplo, han contribuido de forma efectiva en la construcción de la marca, y en el caso específico de la plataforma de cocreación, presenta la ventaja de ser un recurso inagotable como fuente de ideas innovadoras que se pueden continuar potenciando al corto y largo plazo, pues además, estas ideas pueden contribuir con los demás frentes que requieren innovación en la ciudad, de forma que obtenga cada vez mejores mediciones en este tipo de clasificaciones que son indicadores del posicionamiento de la marca-ciudad.

En reconocimiento al gran impacto logrado por la plataforma, MiMedellín recibió en septiembre de 2015 el Premio Interamericano a la Innovación para la Gestión Pública Efectiva otorgado por la OEA. Con este premio, que en esta edición tuvo 218 postulaciones de 16 países, la OEA busca identificar las innovaciones en gestión pública que realizan los distintos gobiernos locales, regionales y nacionales de las Américas, para reconocerlas, sistematizarlas, incentivarlas y promocionarlas como experiencias útiles y posibles de replicar en otras latitudes, lo que ratifica como el impacto de esta iniciativa no solo se ha dado en términos de su contribución al desarrollo de la ciudad y la calidad de vida de sus habitantes, sino que además contribuye al convertirse en un referente de innovación ante el mundo, ligado directamente a la marca-ciudad.

Ahora bien, si se analiza con detenimiento todo el desarrollo de la estrategia de la marca-ciudad con relación a las prácticas de cocreación, es identificable un patrón fundamental que se desarrolla en sinergia con estas, que es el involucramiento de 
múltiples actores para posibilitar el éxito de este tipo de iniciativas. Se evidenció a lo largo de esta investigación, como no únicamente el aporte e interacción entre ciudadanos conlleva a los resultados de las iniciativas, en cambio, se observa que es a medida que junto con ellos intervienen y se suman actores de otros sectores como lo son gobierno, academia, colectivos sociales y empresas, que las propuestas logran desarrollarse, es entonces cuando logran realmente evolucionar de ideas a verdaderas soluciones que contribuyen a mejorar el entorno urbano. Así lo reconoce la corporación Ruta N (2016) en una declaración al final del documental Código Origen donde afirman:

Su éxito -el de Medellín- se debe al entendimiento de que la innovación como proceso no es exclusivo de empresas y universidades, sino también de las organizaciones de todo tipo y también de todas las personas (Archivo de video: https://www.youtube.com/watch?v=_sOq6L2uEIA).

En el mismo sentido, David Sierra, experto en ecosistemas de innovación entrevistado por la Corporación Ruta N, afirmó que si tuviera que encontrarse una definición del éxito que ha tenido Medellín, esta se encuentra en la gran relación que hay entre la universidad, empresa, estado y sociedad (2016).

A esto se le suma la visión expuesta por Rocha (2016), que en su entrevista afirmó que cocreación no se trata simplemente de que la gente opine, pues no es lo mismo crear que opinar, y por lo tanto plataformas como MiMedellín que en principio son espacios de participación, no pueden reducirse a ser centros de opinión, sino que deben poner en contacto a los diferentes actores para que juntos construyan soluciones realmente innovadoras, a partir de las diferentes experiencias, perspectivas profesionales y técnicas diversas, constituyéndose así como verdaderos espacios de cocreación.

\section{CONCLUSIONES}

Se puede concluir entonces que el impacto de la práctica de cocreación en la estrategia de marca-ciudad para el caso de Medellín, no se ha presentado únicamente a partir de la plataforma MiMedellín, sino que esta forma de innovación social es de 
hecho el eje transversal de la toda la estrategia planteada por Ruta $\mathrm{N}$ y que todas las tácticas que ha diseńado, ya sean foros, eventos, laboratorios o plataformas, como MiMedellín, hacen parte del proceso de cocreación de la ciudad en su conjunto, pues están encaminadas a permitir que cada actor aporte desde su propio campo de acción, para lograr la meta de posicionamiento planteada como ciudad innovadora.

Se trata entonces de entender que es justo en ese escenario, donde confluyen perspectivas completamente distintas, que surge la verdadera innovación de la cocreación, pues posibilita un proceso de realimentación mutua que aprovecha el conocimiento colectivo, tanto de la ciudadanía -a través de MiMedellín-, como también de la academia, las empresas y hasta el mismo gobierno local, lo cual permite finalmente potenciar la ideas al máximo y es así como se logra impactar directamente las percepciones de las personas tanto externa como localmente, y se consigue modificar el posicionamiento de manera efectiva a favor de la estrategia planteada. Por eso es vital que todos los involucrados sientan y evidencien una dinámica de escucha activa, que los motive a aportar sus ideas, tal vez gran parte del éxito de Medellín con su estrategia ha sido precisamente este punto, pues hasta para los ciudadanos comunes esta es una ciudad que además de tener en cuenta sus opiniones, les da un valor significativo que se hace visible en la construcción social que edifica la marca.

En este sentido, el impacto particular de la plataforma MiMedellín es tal vez el más valioso, pues contribuye como representante de las perspectivas de una de las figuras centrales, que son los ciudadanos y sus colectivos, encargándose de conectarlos directamente con otros actores como el gobierno local y sus secretarias, de forma que las ideas allí compartidas puedan, una vez son evaluadas, trascender y evolucionar a soluciones urbanas reales, gracias también a la intervención de otras organizaciones -universidades y sector privado- y así, los participantes pueden evidenciar el valor de sus ideas al ser materializadas.

A través de todo este proceso es como finalmente se logra una implementación eficiente de las iniciativas de cocreación dentro de la estrategia de la marca, consiguiendo tener un impacto realmente significativo que aporte de manera positiva, como lo han venido haciendo, a alcanzar el posicionamiento deseado de Medellín como la ciudad 
más innovadora, pues hace que desde la forma como las ideas son recolectadas y concebidas en la plataforma, sea innovadora por sí misma, lo que a su vez redefine y lleva a la realidad el concepto de gobierno abierto, todo en beneficio de los fines de posicionamiento y competitividad planteados desde de la marca-ciudad y por ende también, aporta al desarrollo mismo de la ciudad, impactando la calidad de vida de sus habitantes, brindándoles un entorno donde las oportunidades y el progreso están al alcance de todos.

\section{REFERENCIAS}

Alcaldía de Medellín. (2014). Secretaría de Movilidad lanza su reto en la plataforma MiMedellin: ¿qué solución creativa propones para reducir el uso del vehiculo particular? Recuperado de https:/www.medellin.gov.co/movilidad/component/k2/secretaria-de-movilidad-lanza-su-reto-en-la-plataforma-mi-medellin-que-solucion-creativa-propones-para-reducirel-uso-del-vehiculo-particular

Escorsa, P. (1997). Tecnología e innovación en la empresa. Dirección y gestión. España: Editorial UPC.

Hine, C. (2011). Etnografía virtual. Barcelona: Editorial UOC.

Las salinas. (2015). La cocreación: enriqueciendo los proyectos urbanisticos. [Online]. Recuperado de http://www.lassalinas.cl/blog/posts/la-co-creacion-enriqueciendo-losproyectos-urbanisticos

MiMedellín. (2016). El Compromiso, un barrio innovador de Medellín. [Archivo de video]. Recuperado de https:/www.youtube.com/watch?v=J7saXfLHvW8

Neutralidad de las plataformas. (2014). Fomentar un entorno digital abierto y sostenible. Recuperado de http://cnnumerique.fr/wp-content/uploads/2014/06/Neutralidad-de-lasplataformas-ESP.pdf 
Parente, A. M. (2013, 27 de enero). Innovación y más innovación, ¿qué tal cocreación? Recuperado de http://blogs.portafolio.co/los-aparentes-negocios-internacionales/innovacion -y-mas-innovacion-que-tal-co-creacion/

Prensa CES. (2015). La Universidad CES está invitada a participar del proyecto MiMedellín. Recuperado de http://prensaces.ces.edu.co/bitacora/113-mayo-de-2015/992-la-universidad-ces-está-invitada-a-participar-del-proyecto-mi-medellín.html

Puig, T. (2009). Marca-ciudad. Barcelona: Paidós.

Ramaswamy, V., y Gouillart, F. (2010, 11 de enero). El poder de la cocreación. Recuperado de http:/www.tantum.com/conocimiento/el-poder-de-la-co-creacion/

Ransbeeck, D. V. (2015, 31 de diciembre). 3 key learnings to move forward with citizen engagement \& cocreation. Recuperado de https://medium.com/@DriesVRansbeeck/3key-learnings-to-move-forward-with-citizen-engagement-co-creation-c33b0bdde $3 \mathrm{~d} 2 \#$. tyayooj7d

Rocha, J. (2016). Entrevista a Juan Pablo Rocha. Oficinas de J. Walter Thomson Bogotá-Colombia.

Ruiz, J. I. (2012). Metodología de la investigación cualitativa, (5.a ed.). Bilbao: Deusto.

Ruta N. (2014, 25 de enero). ¿Cómo participar en Comuna Innova? Recuperado de http:// rutanmedellin.org/es/actualidad/item/como-participar-en-comuna-innova-25042014

Ruta N. (2015, 26 de enero). Gracias a los ciudadanos, Laureles se convertirá en el primer barrio bici-amigable. Recuperdo de http:/www.rutanmedellin.org/es/actualidad/item/ laureles-primer-barrio-bici-amigable

Ruta N. (2016). Documental Código Origen. [Archivo de video]. Recuperado de https:// www.youtube.com/watch?v=_sOq6L2uEIA 
Secretaría de movilidad, alcaldía de Medellín. (2014). Obras del tranvía de Ayacucho llegan al centro para transformar nuestra ciudad. Recuperado de https://www.medellin.gov.co/movilidad/component/k2/obras-del-tranvia-de-ayacucho-llegan-al-centro-para-transformar -nuestra-ciudad

Semana.com. (2016). Medellín gana el Nobel de las ciudades. Recuperado de http://www.semana.com/nacion/articulo/medellin-gana-premio-lee-kuan-yew-world-city-2016/465583

Soler, P. (1997). La investigación cualitativa en marketing y publicidad. En Cubí, M. (Ed.). Barcelona: Paidós Ibérica, S. A.

Tinto, J. A. (enero-junio de 2008). La imagen de marca de las ciudades. Recuperado de http:// www.redalyc.org/articulo.oa? $\mathrm{id}=55501905$

Waissbluth, M., Contreras, E., Galaz, P., Aguilera, I., Inostroza, J., Infante, A., Barros, A., Westermeyer, N., Saieg, G., Vidal, F. y Gatica, M. A. (2014, septiembre). Cocreación para la innovación: un caso en el sector público chileno. Revista Ingeniería de Sistemas, 28. Chile. 